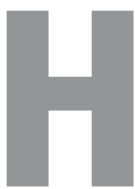

itting the genetic profile button on his computer, David White, a general practitioner, gets a list of the antihypertensive drugs and doses most suited to the patient sitting in front of him. He gives the computer generated prescription to the patient. This is what Francis Collins, leader of the Human Genome Project and director of the US National Human Genome Research Institute, predicted doctors would be doing in the next few years.

In 2001, he wrote: "Genetic prediction of individual risks of disease and responsiveness to drugs will reach the medical mainstream in the next decade or so. The development of designer drugs, based on a genomic approach to targeting molecular pathways that are disrupted in disease, will follow soon after." ${ }^{1}$

This prediction now looks optimistic. "Biology is much more complex," comments Klaus Lindpaintner, head of the Research Center for Medical Genetics with Roche. "But the idea of pharmacogenetics is certainly playing an increasingly important role in the way we think about developing drugs.”

\section{What is pharmacogenetics?}

Pharmacogenetics analyses genetic differences between individuals in their response to medicines. Professor Lindpaintner explains the attraction: "This approach promised more bang for the buck for patients and providers, with the greater likelihood of a response and reduced risk of side effects."

Health service providers are keen to explore genetic approaches to optimising use of drugs. The UK genetics white paper in 2003 identified pharmacogenetics as a research priority. ${ }^{2}$ It said: "Pharmacogenetics will lead to prescribing which is more effectively tailored to the needs of the individual," suggesting that this could reduce the waste associated with having to try out drugs before finding the best one for each patient and significantly cut the risk and cost of adverse drug reactions. The government has allocated $£ 2.5 \mathrm{~m}(€ 3.7 \mathrm{~m} ; \$ 4.9 \mathrm{~m})$ for pharmacogenetic research on existing drugs. The Department of Health is also funding the first university chair in pharmacogenetics, supported by two to three full time researchers.

\section{Drug development}

To what extent are drug companies incorporating pharmacogenetic approaches into developing new drugs? Nadine Cohen, head of pharmacogenetics at Johnson \& Johnson Pharmaceutical Research and Development,
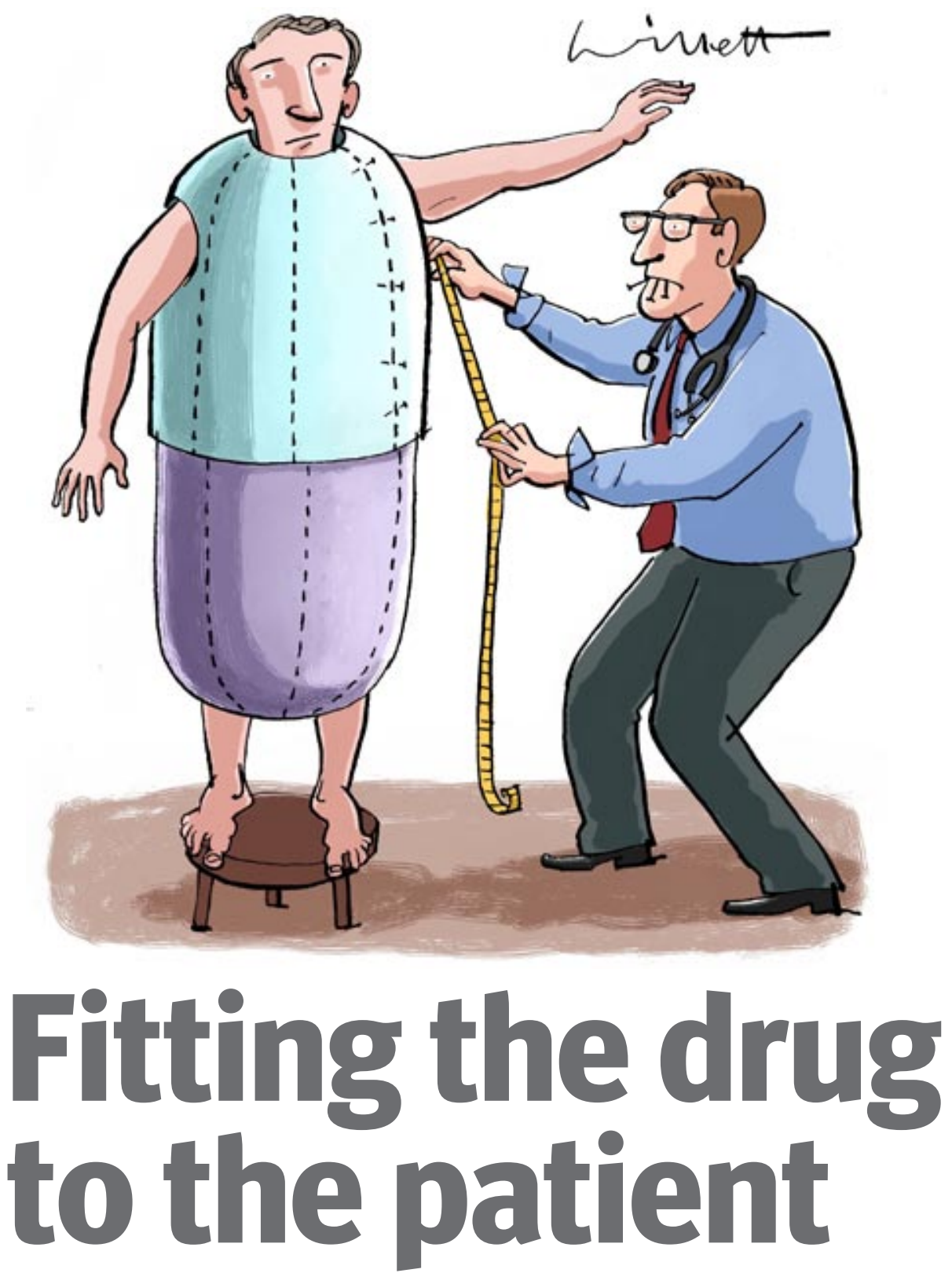

Designer drugs with minimal side effects were meant to revolutionise prescribing. Susan Mayor investigates why the reality may be less dramatic

said: "We need to incorporate pharmacogenetics in research and development as early as possible and include it in all stages from drug discovery through to commercialisation. In drug discovery, we are trying to identify genes associated with disease, which could lead to novel targets, novel diagnostics, and enrich drug development pipelines."

Genetic factors have been considered at an early stage of drug development for several decades in terms of how a drug is excreted, explains Munir Pirmohamed, professor of clinical pharmacology at the University of Liverpool. "In new drug development, if a drug is metabolised by cytochrome $\mathrm{P} 450$ 2D6-a liver enzyme for which mutations are quite common-and other drugs are available, the drug won't be developed further. So drugs are screened out at an early stage, based on pharmacogenetic issues."

The more recent interpretation of pharmacogenetics-prescribing guided by the patient's genotype-is still evolving, according to Professor Pirmohamed. "It is coming along, but not for everything," he said. 
"Where genetic variation between individuals is associated with variations in response to a drug, companies may proceed to look at efficacy and toxicity in that genotype-guided group." This results in genotype guided indication at licensing.

Duncan McHale, head of molecular profiling at Pfizer UK, agrees that pharmacogenetics is being introduced into drug development. "But it is certainly not the panacea suggested in early claims of its potential impact. It has not revolutionised drug development." He thinks that this is unsurprising in such a tightly regulated environment, where new developments have to be explored fully before being tested in people.

\section{Current applications}

Despite a lot of research in the field, relatively few drugs based on pharmacogenetic research have made it to the bedside. Oncology is the most advanced in its use of pharmacogenetic approaches. Greater understanding of the biology of many cancers has provided clear molecular targets for new drugs. And because cancers involve genetic mutations, exploring these is an obvious route for drug development.

Trastuzumab (Herceptin) is the most well known drug to result from pharmacogenomic design. It is prescribed for a genetic variant of breast cancer rather than a genetic variant of the patient, targeting the Her-2 protein. Patients are tested for the protein, not a genetic marker. Another example is imatinib. This inhibits the enzyme Bcr-Abl kinase produced as a consequence of the translocation between chromosomes 9 and 22 that occurs in chronic myeloid leukaemia. Use of genetic approaches is extending to other diseases as more about their aetiology is understood.

Pharmacogenetics is also beginning to have a role in prescription of the reverse transcriptase inhibitor abacavir to patients with HIV infection. Patients with the HLA-B*5701 allele are at greater risk of a hypersensitivity reaction to the drug. Recent studies have shown that the frequency of hypersensitivity falls from more than $5 \%$ to less than $1 \%$ if the drug is prescribed according to HLA typing, according to Professor Pirmohamed. Some centres are now routinely typing patients before prescribing abacavir. He notes: "This is an important example of pharmacogenetics that has worked. It has progressed quickly to clinical practice, only taking four years from identification of the genetic factor involved."

There has also been interest in reviewing pharmacogenetic aspects of drugs already in clinical use. Warfarin is the most advanced in this respect, with two genetic variants in cytochrome $\mathrm{P} 450$ (the enzyme that metabolises warfarin) and vitamin $\mathrm{K}$ epoxide reductase (the target for the drug). "Looking at these two markers, together with age and body weight, gives a good degree of precision in dose requirement," said Professor Pirmohamed. However, further studies are needed to assess whether they would be sufficiently accurate for clinical practice.

\section{Niche markets?}

Have fears that drugs would be niched by pharmacogenetics, greatly restricting potential markets, put companies off taking this approach? Roland Wolf, director of the biomedical research centre at Dundee University, thinks not: "There was concern about restricting markets by subdividing into genetic groups. But when you develop new drugs, you want to optimise the potential for a drug to succeed in phase III trials. If you can identify genetic variance that may define differences in the way the drug works, you would be crazy not to carry out genetic testing as part of clinical trial." $\mathrm{He}$ points out that if a genetic variant meant that only $20 \%$ of the population responds to a new drug, a trial in the entire population might not show an advantage over an existing treatment.

Pharmacogenetics is just one approach that drug companies are using to develop new treatments, says Dr McHale. "We see pharmacogenetics as one of a range of approaches that we will use-along with transcription analysis and proteomics." He thinks that for some drugs pharmacogenetics is key in defining the risk:benefit ratio. "But this is unnecessary for other drugs that work well and are well tolerated in the majority of people."

Professor Lindpaintner agrees: "We kid ourselves if we think there are responders and non-responders; there are only people who respond more or less." He warns that finding indicators that accurately predict likely responders is very challenging: "The last thing we want to do is inappropriately withhold a potentially beneficial medicine. You need a marker with high specificity and sensitivity-around 75-80\%-before you can use it in diagnostic research."

He thinks that the genotype might be the wrong place to look for biomarkers of response to most drugs. "DNA sequences are not known aside from a few rare diseases. And DNA is the most remote level from where life happens, which is at the level of proteins and small molecules. Therefore, we are more likely to see diagnostic tools at the level of protein expression."

\section{Way forward}

Most drug companies have developed inhouse expertise in genetics, but collaborations with academic research groups and biotech companies are also flourishing. One example is a serious adverse events consortium that is currently being formed as a partnership between the US Food and Drugs Agency and drug industry.

Looking to the future, predictions are much more cautious than those made a few years ago by Dr Collins. Professor Lindpaintner believes "pharmacogenetics is here to stay but we need to be realistic about its potential, which is limited given the highly complex aetiology of common diseases. They are not single gene disorders. Most are multifactorial, with genetic and environmental factors."

Dr Wolf sees another reason why genetic approaches may not be the holy grail of new drug development. "Drugs with a wide therapeutic index won't show much variability in response, so there is not much point in exploring the pharmacogenetics. For diseases with a readily identifiable marker-such as blood pressure for hypertension-you might as well give the drug according to blood pressure, so can do without genetics."

"We have moved into an era of realism rather than hype in the potential of pharmacogenetics in new drug development," concludes Professor Pirmohamed. "Genetic aspects have to be looked at in association with other factors, including environment, in the clinical use of drugs. Just because a drug doesn't work in a patient doesn't indicate genetic variation in response is the cause. The patient may just not be taking the drug. Genetics are just part of how patients respond to drugs."

Susan Mayor, freelance journalist, London susan@mayor.dircon.co.uk

Competing interests: SM has received payment for writing and communications projects from medical charities, government organisations, publishing companies, drug companies, and communications agencies.

Collins FS, McKusickVA. Implications of the human genome project for medical science. JAMA 2001;285:540-4.

2 Department of Health. Our inheritance, our future: realising the potential of genetics in the NHS. London: DoH. 2003 www.dh.gov.uk assetRoot/04/01/92/39/04019239.pdi 